\title{
Thought-Forms Gone Rogue: A Theory for Psi-Critics and Parapsychologists
}

\author{
Adrian PARker \\ Department of Psychology, University of Gothenburg, Sweden \\ Adrian.Parker@psy.gu.se
}

Submitted July 16, 2020; Accepted September 26, 2020; Published March 15, 2021

https://10.31275/20211901

Creative Commons License CC-BY-NC

\begin{abstract}
It is argued that psi-critics Reber and Alcock have lifted the debate from the impasse concerning the evidence for the existence of psi phenomena, toward focusing on understanding the nature of the phenomena. This focus concerns the demand to show that statistical findings are not anomalies but reflect real cause-and-effect relationships and to find a common theoretical framework for what otherwise appear to be heterogeneous rogue phenomena. It is maintained here that the demand for showing causal relationships is already met by a methodology using real-time recordings of changing target imagery along with receiver mentation. The demand by critics for a theoretical understanding linking all or most of the rogue phenomena, led to the proposition advanced here concerning thought-forms and co-conscious states. According to this, the many "rogue phenomena" both in psychology and parapsychology (such as automatic writing, lucid dream characters, spirit possessions, and entity experiences in psychedelic states) are to be understood as representing dissociated thought-forms with varying degrees of coconsciousness and in some cases the development of a genuine degree of autonomy and identity.

Keywords: altered states, thought-forms, consciousness, psi, skepticism, automatic writing, co-consciousness, possession
\end{abstract}

A major issue impeding the acceptance of parapsychology is that there is no theoretical conceptual framework linking its diverse phenomena. This paper will argue that if consciousness is seen as 
primary in nature and if dissociated states are a normal characteristic of consciousness, then most of the phenomena do form a meaningful relationship. Many of the apparently heterogeneous phenomena in psychology as well as in parapsychology such as automatic writing, lucid dream characters, spirit possessions, and entity experiences can then be understood as representing dissociated thought-forms with varying degrees of co-consciousness and in some cases with the development of a genuine degree of autonomy.

The importance of establishing such a theoretical framework is highlighted by the current critique of parapsychology as a field of science by Reber \& Alcock (2020) (R \&A), although this critique by R \& A was actually based on three grounds. These are 1 ) that the phenomena contradict the limiting principles on which science is based, 2) that the claimed psi (paranormal) effects have small effect sizes without any shown causal relationship to reality, and 3) that there is no theoretical conceptual framework that brings order to the chaos in the range of alleged phenomena. The first objection from $R \& A$ was met in the 2019 issue 4 of the Journal of Scientific Exploration by an Editorial and five individual author responses, all making counterarguments to what seemed to be R \& A's assertion that psi phenomena are "impossible" because they contradict the limiting principles of science, which have served science so well that they could almost be written in stone.

The second objection by $\mathrm{R} \& \mathrm{~A}$ claiming that there were only small effect sizes in parapsychology was partly met by showing their equivalence to those of other psychological findings (Williams, 2019), but the final thrust of R \& A's second argument—that the "Statistical departures from chance expectation cannot and do not identify the causes of those departures" (Reber \& Alcock, 2020, p. 397)—remains unparried. Since this is an issue that has been of some concern in our work at Gothenburg, some space will be given to this before proceeding to the related issue of the theoretical framework for understanding the phenomena.

This challenge assumes that all the significant experimental findings concerning ESP may only represent unknown artifacts or "Error Some Place" rather than causal relationships. This challenge is met if it can be shown that highly significant scores relate closely and apparently causally to the content of the experiences behind the scores. 
The first attempt to deal with this occurred in some of the early ganzfeld work where the descriptions from successes in the telepathy setup were presented as voice-overs and were shown to closely match the target film clips (Honorton et al., 1990). However, there remained a possible bias in making this selection since there was no check on whether or not the recording of the receivers' mentation reports actually matched in real time the content of senders' film clips. Without this check, the matching could be seen as rather arbitrary. The series of real-time experiments we carried out at Gothenburg rectified this, and in doing so they deal directly with the critique of $R$ \& A concerning the lack of connection to reality.

Indeed, the very purpose of our real-time recordings with the digital ganzfeld was to produce the laboratory analogue of spontaneous psychic experiences where one person (the receiver) has a psychic experience in an altered state, usually a dream-which corresponds in content and in time with another crisis experience of another person (the sender). In the laboratory setup this meant that we would eavesdrop on the person in the altered state (the ganzfeld state) describing in the successful cases the sender's experience of an emotionally loaded film clip. The arrangement enabled us to record both experiences precisely as they were happening and to highlight the apparent causal relationship (Parker et al., 2000). These hits occurred when the participants not only chose the correct clip but their descriptions seemed to closely and causally follow unpredictable changes in the film content. About one in six of our real-time (first rank) hits were of this type. Coincidental false matches did occasionally occur but these matches did not seem to show continuity in following the unexpected changes. This was a distinctive feature of genuine matches, but because of a flaw in the choice of data used for correctly accessing this, the final evidence is still lacking (see Parker, 2020, for a further discussion.)

An ideal opportunity for dealing with R \& A's challenge, however, did arise during the ganzfeld testing of a consistently successful motherdaughter pair. I was curious to know if an unexpected intervention in the "sender" room, where the daughter was situated and observing a film clip, would intervene in the flow of ganzfeld mentation of the mother located in the "receiver" room. Without informing any of the participants prior to the session of my intention, I asked the daughter 
to leave the room and a close friend of the mother to come into the room and take over the role of sender. At precisely the point in time when the friend entered the room, the mother's flow of mentation images was interrupted and her voice was heard from the receiver room and recorded on tape saying "Where have you been?" This was the only occasion when I attempted such an intervention that fits with $R$ \& A's search for the causal effects, but others occurred spontaneously such as when the tape ran out unexpectedly or the film went into slow motion when the mentation responses were the words "change tape" and "slow motion."

The real-time digital ganzfeld does not stand alone in relating statistical findings to reality events. The work of James Carpenter (2012) has been outstanding in this respect, and on one occasion even used majority voting to successfully "transmit" the code-word peace (Carpenter, 1991).

I suspect that R \& A would prefer to call these examples merely weird synchronous events, in which case I would agree with the terminology. Rather than extrasensory perception, a better term for ESP may indeed be Extraordinary Synchronous Phenomena. (The alert reader may ask about precognition, but this would involve a discussion of the specious presence. Although this lies beyond the scope of this article, it forms a part of Carr's theory [Carr, 2019]).

The accumulation of more evidence for psi has become a Sisyphean boulder for those wishing to resolve this issue. If the boulder is now to be moved forward, we still need to deal with the third objection.

The third objection concerns the maelstrom of phenomena, or as R \& A prefer to call it "the farrago." They write: "In contemporary parapsychology one finds a crazy quilt set of effects that have no conceivable underlying foundation. It is as if actors from a dozen different plays have appeared on the same stage in a discordant farrago" (Reber \& Alcock, 2020, p. 393.)

I agree with R \& A. This issue is in dire need of being resolved. To do so, we need to state not only how the phenomena relate to each other but also how they relate to other known natural phenomena. Low-level hypotheses do already exist, as pointed out in two of the responses to R \& A (Roe, 2019; Williams, 2019). These hypotheses include the relationship to noise-reduction (Honorton, 1977; Storm et al., 2010), to 
top-down processes (Parker, 2000), to change-in-state (Murphy, 1966; Honorton, 1977; Parker, 1975, 1994), to unconscious expectancies and belief systems (Carpenter, 2012; Parker et al., 1997), and to morphic fields (Sheldrake, 2020). Although I have been active in formulating and testing some of these hypotheses, I freely admit these do not provide any commonality that would link the various paranormal phenomena, and they give neither a proper theory nor a deep understanding of what we are dealing with.

The demand to relate paranormal phenomena to known natural processes can be understood when the role of science from the Renaissance onward is seen as being "the candle in the dark." Science has created a secure, and largely predictable, orderly world, and followers of reductionistic science have little or zero tolerance for ambiguity or any retreat to the darkness of the occult. Rogue and occult phenomena can be seen as a threat to this Weltanschauung.

Perhaps this is why some parapsychologists with a background in physics disavow the existence of any phenomena other than ESP or even prefer "anomalous cognition," since this allows them to pin their hopes on the nonlocal effects discovered in quantum physics, where a connectedness, analogous to telepathy, occurs between particles at various distances. In addition, the duality of light waves and particles seems to demand an observer, that is consciousness, in determining the final outcome of these processes (Marwaha \& May, 2015a; Millar in Parker \& Millar, 2014; Stapp, 2015). Others would go further and argue that quantum theory requires the presence of consciousness in order to explain the transition to reality. In making these assertions, parapsychologists often cite the writings of eminent physicists such as Max Planck, Eugene Wigner, and Werner Heisenberg, who all endorsed the view that consciousness is a primary mover in the universe (Radin, 2013, 2018; van Lommel, 2020).

Unfortunately, this argument is seldom well-received given that the majority of modern physicists appear not to share the view that consciousness is involved in quantum outcomes (Carr 2019; Mroczkowski \& Malozemoff, 2019), although it may be more accurate to say that such a view is rather controversial (Kastrup, 2019). What seems clear is that most physicists agree the bridge has not been found that would link phenomena occurring at the quantum level to those occurring in everyday 
human reality-the so-called measurement problem (Carr, 2019).

Against this background, appeals to the various interpretations of quantum theory can only strengthen any natural aversion the skeptic might have against ambiguity. Arguments about the similarity of nonlocal effects between particles and telepathy between humans, are seen as simply attempting to explain one unknown in terms of another unknown.

Here I will argue that the opposite tactic is more forthright and effective. What is surely needed is an alternative view of psi that enables us not only to explain the findings of ESP experiments but also the range of rogue phenomena in parapsychology from poltergeist cases to alleged spirit communications of mediums. I will further argue that we need to relate these rogue phenomena to an even wider range of phenomena such as automatic writing, and to savant abilities, which are a perennial challenge to clinical psychology and psychiatry. The situation is particularly favorable for such an approach given that consciousness is now a legitimate subject for scientific study. Support for such a starting point is found not only in the writings of the exceptional physicists named above but also in those of a few exceptional psychologist-philosophers such as William James and Frederic Myers who also insisted that consciousness is primary in nature (Kelly et al., 2007).

The insistence that consciousness is primary, is of course the idealist version of the panpsychism equivalent of the "one-free miracle" required by reductionism as parodied by Terence McKenna: "Give us one free miracle and we'll explain the rest. The one free miracle is the appearance of all the mass and energy in the universe and all the laws that govern it in a single instant from nothing" (McKenna, in a personal communication with Rupert Sheldrake in 1985). The difference here with panpsychism is that empirical work in parapsychology indicates that this one miracle, in this case consciousness with its attribute of psi, does occur.

Seen from this perspective, psi is merely a sign of the fundamental connectedness of consciousness, and physics would then only need to enter the picture as a way for consciousness to explain nature. This connectedness is most noticeable in altered states of consciousness as illustrated by the cases reported in the spontaneous literature from 
real life and confirmed in the laboratory by ganzfeld and altered-state research (Storm et al., 2010).

Of course, if we are to escape the chaos of R \& A's "farrago," then we need to find some further commonality between laboratory ESP and the diversity of experiences such as spirit communications through mediums, mystical experiences, apparitional experiences, and poltergeist phenomena. In seeking this commonality by referring to the concept of "thought-forms," it is not being claimed that this provides the ultimate sought-after deeper explanation. In order to seek this, it may well be necessary to integrate the concept further into the framework of a theory from modern physics but one that gives primacy to a dimension of consciousness in describing the natural world (e.g., Carr, 2019; Pilotti, 2011).

\section{THOUGHT-FORMS}

A thought-form has been defined as "any perceivable form, which has been created directly and exclusively by the mind, unconsciously or consciously, and which in some cases develops autonomy" (Parker \& Puhle, 2018). If we follow this definition, it is described in many different cultural contexts; the most well-known examples being the Celtic and Germanic "pooka," the Arabian "djinn," and the Tibetan "tulpa." The pooka is most well-known through the works of Shakespeare, while the tulpa is attributed to Tibetan beliefs popularized by Alexandra DavidNéel. David-Néel, while travelling in the Himalayas, claimed to have produced a tulpa, with its own will. However, according to a recent paper, this concept is a combination of theosophical beliefs and a somewhat misappropriated Tibetan concept (Mikles \& Laycock, 2015).

Nevertheless, what the concept of thought-forms brings to the debate forum, is to view consciousness as non-unitary and potentially dissociative and to recognize that this characteristic has various cultural expressions. There is in fact a commonality to all this if we presuppose that there is a transpersonal or 'extended level to consciousness,' which is capable of creating entities in response to extreme personal needs. Assuming this occurs, these thought-forms could in some states of consciousness eventually develop their own sense of identity, interact with other persons in a meaningful way, and in some extreme cases express their own will. 
As preposterous as this may seem, there is actually consistency in modern research concerning these aspects. As I shall now present in detail, these apparently autonomous entities are reported as occurring in a variety of altered states such as lucid dreaming (Johnson, 2017; Puhle \& Parker, 2017; Tholey, 1989; Waggoner \& McCready, 2015); psychedelic experiences (Luke, 2012); and near-death-experiences (E. W. Kelly, 2001). In addition, it is being proposed here that the communicating spirits of mediums can be explained as autonomous thought-forms gaining the identity of deceased individuals. This means that in some cases they are not secondary personalities or appended "alters" but have become independently conscious alters.

\section{Lucid Dreaming}

Lucid dreams are dreams in which the dreamer retains the critical ability to realize that what is being experienced is a dream world and continues dreaming while maintaining this awareness. About $60-80 \%$ of individuals report having these experiences and about $20 \%$ have them on a regular basis-a least once a month (Schredl \& Erlacher, 2004). Some individuals become adept at this and develop the ability to maintain the states for longer periods and to steer the content and direction of their dreams. Although lacking exact figures, it appears clear that the majority of the adepts report experiencing so-called dream characters.

A way of understanding dream characters is to relate them to the concept of dissociation. Dissociation is defined as "one or more parallel paths or systems operating outside of awareness and influencing cognition, affect, or behavior" (Kirsch \& Council, 1992, p. 275). The work of Harvard clinical psychologist Deirdre Barrett (1994a; 1994b; 1995; 1996) is crucial here because it links dissociation to dreaming with the starting point that "each night when we dream we manufacture others out of parts of ourselves" (1994a, p. 123). In principle, this is the same process of dissociation that individuals with "Dissociative Identity Disorder" use in creating their "alters." A confusion can occur in such cases between what occurred in dream life and in waking life as well as in sorting out the experiences that belonged to the alter and to the host personality. To clarify this, Barrett carried out a questionnaire study of the dream experiences reported by such individuals, which indicated 
that 13 of the 23 patients experienced their "alters" not only in real life but also as dream characters. In addition, eight of these patients had personalities reporting different dream memories. This led Barrett to conclude that dream characters are "normal dissociative cognitive and personality processes which operate largely outside consciousness." In waking life these characters can occasionally break into consciousness as dissociations (Barrett, 1995, p. 66). According to this perspective, lucid dream characters represent unexpressed parts of individuals that gained, so to speak, their own consciousness and are a normal analog of alters.

Indeed, many of the entities or characters appearing in lucid dream states do assert that they have their own independent memories or identities. Paul Tholey, a pioneer researcher of lucid dreaming, reported that when one of the characters was questioned in the lucid dream about his true identity, the character responded with: "I am sure that I have a consciousness, but I doubt if you have one, because you ask me such stupid questions!" (Tholey, 1989, p. 574). In another account, a dream character asserted "if I am a dream character then how come I can recall a whole other life before this moment. I have a husband and child and a whole other life" (quoted in Waggoner \& McCready, 2015, p. 105).

Since Tholey's time onward, most lucid dream researchers fall into two groups. The first group are university-based researchers who publish in mainstream journals and focus on the normal characteristics and the psychophysiology of lucid dreamers rather than on the extraordinary experiences of the so-called adepts. The second group are themselves adepts, sometimes with an academic education, who have taken it upon themselves to document and explore their own experiences and those of others. It is of course easy to dismiss the accounts from this latter group as uncritical and biased, but perhaps a fairer critique is that many authors support their personal records with those taken from Internet sources. Nevertheless, one assertion in these accounts is currently being confirmed by our ongoing study of adept lucid dreamers: The adepts in our study do report a remarkable ability to exert control over the induction of lucid dreaming, to explore its content in a critical way, and to experience dream characters.

An example of these authors is Robert Waggoner. Although 
concluding that most dream characters represent previously unexpressed sides of oneself, Waggoner asserts that this is not always so since his efforts to dismiss the characters occasionally result in the attitude described above by Tholey: "Some dream figures remain and look at me with a sense of disbelief that I did not recognize their independence from my thought processes" (Waggoner \& McCready, 2015, p. 110). Indeed, this does seem to be the consensus opinion among the adepts. Clare Johnson, another adept who is an academic writer, suggests that lucid dream characters can vary from passive wooden characters to those expressing psychological needs, and ultimately take the form of what appears to be a spiritual guide. In agreement with Waggoner and Johnson, David Jay Brown, who has a neuroscience background, sees characters as creations of his own mind, but also believes that some characters may belong to other minds, and others may be even be "higher beings" (Brown, 2016).

One of the few research psychologists to study dream characters is Tadas Stumbrys. On the basis of his online survey, it would seem that about half of dream characters are friendly while one fifth are hostile (Stumbrys \& Erlacher, 2017). As compared to those suffering from nightmares, the adept lucid dreamers were able in some way to resolve the conflicts with the hostile figures, suggesting some dream characters may express intra-psychic conflicts. Another study by Stumbrys and co-workers (Stumbrys \& Daniels, 2010) indicated that dream characters show a high degree of creativity and cognitive functioning, but, interestingly, yet another study (Stumbrys et al., 2011) confirmed Tholey's observation that their mathematical ability was limited-to that of primary school children.

Adding to the view that lucid dream characters are dissociated parts of cognition and personality, are the accounts of the lucid dream figures indicating that they have their own visual perspective, and even their own thinking (Tholey translated in Johnson, 2017, pp. 117, 124). Among the anecdotal reports from lucid dreamers who described meeting deceased dream characters, were also about ten percent claiming to have received information they could not have known (Puhle \& Parker, 2017).

Besides the need for more objective independent research, what is obviously lacking in the current research into these experiences is an 
attempt to distinguish between reports relating the different types of lucid dream figures as categorized by Waggoner, Brown, and Johnson.

\section{Near Death Experiences (NDEs)}

A major survey of cases of NDEs from the USA, Canada, and Australia found that $69 \%$ of the collected cases reported the presence of someone, and in $10 \%$ of cases they saw and recognized the deceased person (E. W. Kelly, 2001). Only $4 \%$, eleven of the 274 cases, reported seeing living persons whom they recognized. There was no clear relationship of seeing deceased persons with the medical condition or closeness to death of the person concerned. The perception of these figures seems to be a core feature of the NDE since those who saw deceased persons were more likely to report the other major features of NDEs-enveloping light, an OBE, darkness, or a tunnel-like experience. In seeking "normal" explanations, it remains an unanswered question as to how many of these figures are created by dissociation and how many by mere expectancy. Whatever the case is, they do also fit with "End of Life Experiences" where caretakers and relatives frequently report dying persons having experiences with apparitions coming to "take them away" and deathbed visions (Fenwick et al., 2009).

Because NDEs are often dismissed as hallucinations caused by expectancies and dissociation, the many claims of veridical perception of near-death experiences have become contentious (see Holden et al., 2009, and Rivas et al., 2016, for reviews of these cases). Each of these cases has been subjected to careful scrutiny in attempts to identify alternative explanations-often without much respect for their plausibility. However, if it is conceded that ESP in the laboratory has been established and that it occurs most often during altered states of consciousness, then it is quite conceivable that ESP is an integral part of consciousness in NDEs. This still begs the question as to what ESP actually is, and what is the nature of the experience as a whole?

Michael Nahm (2011) has provided one answer to this in his impressively comprehensive survey of NDEs where he focuses on cases that can be described as "reciprocally confirmed." By reciprocally confirmed, he means the information encountered during the NDEs has been independently confirmed by information found in related 
coincidental cases. An example of this would be from medium communications where the dead person announces a birth and the born child later recalls the previous existence. Another example would be where the person during an NDE sees a living person and that living person experiences an apparition of the other person having the NDE. Other apparently confirmatory cases concern shared NDEs, and intermediate states between death and reincarnation.

In finding such albeit extraordinary cases, Nahm argues that a full circle seems to have been created in tracing the continuation of consciousness after death from NDEs, intermission, and reincarnation. However, the cases Nahm reviews vary greatly in evidential value and he makes no attempt to evaluate their quality.

\section{Automatic Writing and Savant Abilities}

Automatic writing is an enigmatic form of dissociation cases where self-proclaimed entities assert their own independent identity and sometimes demonstrate skills that the normal personality has never, as far as can be determined, had the opportunity to acquire. One of the most outstanding cases is that of Patience Worth who claimed to be a 17th-century Englishwoman who had emigrated to and died in the United States. A proficient knowledge of the Anglo-Saxon language and its culture was demonstrated by the host personality, Pearl Curran, a thirty-year-old and a school dropout who had never left the Midwest. What was equally astounding was the incredibly rapid production of words and images, which spontaneously entered Curran's mind without any conscious effort or control. This suggests Curran had developed a form of co-consciousness. Co-consciousness is in terms of modern psychology represented as multitasking, but the form and content expressed by Pearl Curran is clearly exceptional. Moreover, the actual source of the information produced by Curran remains unexplained.

The case was investigated by W. F. Prince (1927/1964). Reputed to be critical-minded, Prince rejected the spiritualistic claims made by Patience Worth but was unable to offer any plausible explanation for her behavior. In recent years Stephen Braude (2003) devoted considerable time and effort to the case and even conducted further searches, which put to rest any notion that a real Patience Worth had ever existed. Braude was then left with the theory that Patience Worth was an "alter" 
whose presence allowed the latent literary skills of Pearl Curran to gain expression. Yet, there was never any evidence of Pearl Curran in earlier life having practiced these skills and nor had she shown any dissociative states prior to the entry of Patience. On the contrary, Pearl had regarded the Ouija board as producing "silly chatter" and did not seem motivated to acquire any skills. A more recent review of the case by Gioia Diliberto (2010) concluded much the same as Braude: "The 17th-century spinster gave Pearl's life shape and meaning and allowed her to project herself beyond the confines of domestic womanhood to become a writer." The difference was that Braude did not hesitate to credit Pearl Curran via Patience with a psychic ability to access archaic forms of English and history.

It is of course easy to try to make the Patience Worth case vanish with the magic word "anomaly," but several other cases exist such as Hélène Smith and Rosemary Brown (Braude, 2003). Not only do these cases exist but there is another set of "anomalies": the congenital and acquired savants. In terms of latent abilities, these cases merge with the cases of automatic writing except that their remarkable abilities are not attributed to spirits or secondary personalities but are left hanging in the air, largely unexplained. The majority are congenital cases where severe brain damage has occurred. The genius-like abilities that characterize savants are assumed to have occurred in a compensatory manner following the release of an area of the brain, usually the right hemisphere, from the inhibition which would have otherwise been exerted had it not been for the damage. Yet the challenge, which mainstream psychology has never even attempted to deal with, concerns the savants who know things they never have learned. Darold Treffert, who is generally regarded as the world's leading expert on savant cases, attempts to deal with this by assuming this knowledge must somehow have been "factory installed" into the genetic software of our brains. It would seem to be almost in desperation that Treffert further defines this as a genetic form of the Jungian unconscious or brain plasticity bringing this about (Treffert, 2000, 2017). Yet genetic theories and brain plasticity become rather untenable when we consider the acquired savant cases.

The acquired savant cases generally occur as an outcome of concussion, and so-called "calendar calculators" (who can give the day 
of the week for a date in a given year apparently without calculating) are the most well-known examples of these. There are, however, cases with other skills such as Derek Amalo, described by Treffert, who had never played the piano but who could spontaneously play at a professional level. Most challenging are the cases that can occur entirely devoid of any injury. The skill then occurs almost as a form of epiphany where a detailed knowledge is revealed of, say, the underlying principles of music, art, or math. Treffert even includes in this category some cases of foreign language syndrome in which there was little or no opportunity to have learned the language now fluently spoken (Treffert, 2010, 2017).

As for the explanation in the form of a genetically installed memory, Larry Dossey rightly asks concerning some of these skills (such as calendar calculating) "what is the survival value of knowing, as do savants, vast information in a narrow field that is utterly trivial?" Savants are surely then an example of further "rogue phenomena," but in this case belonging primarily to psychology rather than parapsychology. Nevertheless, they too are shunted aside as anomalies from mainstream clinical psychology and psychiatry. Dossey dares then to express the challenge that savants pose: "Savants are earthquakes that shove sacrosanct theories of the mind off their foundations and reduce them to rubble" (Dossey, 2012).

Perhaps the person who is at least potentially gifted with the insight to resolve some of the enigma, is Daniel Tammet. Tammet is a savant in mathematical and language areas with a diagnosis of Asperger syndrome, although he has become a socially skilled and insightful person. In contributing to understanding just how his abilities work, Tammet describes how his own synesthesia is an integral part of the ability that enables him to see symbols depicted as part of landscapes. Tammet does not calculate in conventional ways; instead the answers come spontaneously and take the form of landscapes that he can identify. His language ability seems facilitated also by synesthesia and by his ability to see connections between words (Parker, 2011).

The logical hypothesis, even if unpalatable for the skeptic, is that the savant phenomena might indicate that consciousness has access to some form of reservoir of knowledge beyond what is registered by normal brains. Indeed, Treffert and others describe that the savant is not learning knowledge but discovering it. The question naturally arises 
then if such savants show any evidence of psi in specific terms. There are a few anecdotal accounts (Rimland cited in Treffert, 2009) suggesting this might be so, but to my knowledge no controlled research has ever examined this possibility.

\section{Psychedelic States}

As is the case with psi experiences, there exists a stark polarization of opinion concerning the reality behind psychedelic experiences, which has inevitably led to a similar entrenchment of positions and unjustified stagnation of research on this issue. Those who have had profound experiences under DMT or LSD tend to be emphatic that they are "more real than anything ever experienced" (Luke, 2017; Cott \& Rock, 2008). Those who have not had these experiences tend to be dismissive of them as chemically caused hallucinations. Rick Strassman (2001), who carried out the first studies following the era of the prohibition of psychedelic research, documented how many of his research participants unexpectedly reported the spiritual value of DMT. The spiritual aspects led Cott and Rock (2008) to find points of commonality between psychedelic experiences and NDEs, and David Jay Brown (2016) listed twenty correspondences between lucid dreaming and psychedelic experiences. What does, however, distinguish psychedelic experiences, especially those of DMT, from other altered states, is not just the spiritual beings and guides that are more frequently encountered, but the vast diversity of alien entities and mythological creatures that are reported.

For those experiencing these weird beings and events, the crucial persuasive factor, reminiscent of the claims made in other altered states, is that these entities, whether they be spirits, elves, dwarves, or reptile-like creatures, give to the perceiver every indication of being sentient beings. For those not having this type of experience, this will naturally just seem ludicrous. Whatever the case, it is still claimed that the "beings" communicate insightful information to those perceiving them (Luke, 2017). It should also be said that David Luke, who is the indisputable expert on this topic, did carry out a review of the research attempting to explain psychedelic experiences in neurochemical terms (as DMT-metabolites, ketamine, and dopamine), but he found no definite causal link. There is considerable evidence, also reviewed by 
Luke (2017), suggesting there is a link between psychedelics and psi experiences-with the caveat that there are special methodological issues in working with psychedelics-issues which so far preclude any firm conclusions.

In terms of explanations, the choice still remains between attributing these experiences to some form of Jungian archetypal unconsciousness released by the specific nature of the psychedelic substance or to a delusionary drug-induced state (or perhaps some combination of both). One can only agree with Luke that only further parapsychological experimentation, carefully adapted to the demands of the psychedelic situation, can take this area forward. Such a study could determine if there occurs an overlap in experiences of suitably chosen individuals who are having simultaneous psychedelic experiences in different locations. In addition to supportive guidance, if a shared phenomenological world were to be experienced, a parapsychological aspect could then be introduced by giving one of the individuals a code or gesture to convey to the other. A shared phenomenological world can apparently also be experienced in shared lucid dreams (Waggoner \& McCready, 2015). At Gothenburg with the collaboration of Engelbart Winkler, we used two Lucia stroboscopes to induce dream-like states in a small group of selected volunteers, but to our chagrin the only success we obtained were from the experimenters when they were in the role of participants (Parker, 2017).

\section{Mediumship}

It is clearly beyond the scope of this review to evaluate the evidence for a postmortem continuation of consciousness obtained through mediums. What is being suggested here is that some of the most convincing or challenging communications from mediums can be understood in the same manner as that for entities appearing during lucid dreaming and psychedelic states, that is as expressions of dissociated and autonomous states. This conceptualization differs in a subtle but important way from the more dismissive hypothesis claiming that these communicators are mere secondary personalities of mediums. The entity is re-conceptualized here, not so much as being a repressed part of the medium personality, but as part of the medium's consciousness that has gained its own integrity and independence. 
Indeed, it is striking how in a manner similar to what has been encountered with lucid dream characters, the communicating entity asserts it has the integrity and willpower of a real person. A muchquoted example is the following message from the entity claiming to be the post-mortem Frederic Myers communicating through the mediumship of Trix Fleming (aka Mrs Holland), the sister of Rudyard Kipling, and one of the principal mediums involved in the "cross correspondences." These were a series of apparently interlocking messages that some contemporaries came to believe Myers and other deceased leaders of the Society for Psychical Research had provided as evidence for survival after death. They were given largely independently by different mediums in various parts of the world as evidence for their postmortem existence. The often-quoted message from the deceased "Myers" reads:

It is impossible for me to know how much of what I send reaches you. I feel as if I had presented my credentials-reiterated the proofs of my identity in a wearisomely repetitive manner. The nearest simile I can find to express the difficulty of sending a message is that I appear to be standing behind a sheet of frosted glass, which blurs sight and deadens sound, dictating feebly to a reluctant and somewhat obtuse secretary. A feeling of terrible impotence burdens me. (Johnson, 1908-1909, p. 208)

The cross-correspondences continued until 1930 but partly because of their complexity, critical evaluation is very divided as to their interpretation (Carter, 2012; Hamilton, 2017; Parker, 2010a). Nevertheless, irrespective of this lack of agreement, the emotional tone and appeal is very evident throughout many of the accounts and is reminiscent of similar appeals by entities occurring in lucid dreams and other states. Previously, the skeptical position has simply dismissed the content of such messages as hallucinations, and that any meaning linking the messages would be due to chance and confirmation bias (subjective validation). That aside, the idea being advanced here is that communicating entities or parts of the original consciousness have an integrity in the sense of being convinced themselves of their own existence and independence.

Just how independent these entities were, is of course part of 
the perennial debate, but it seems as far as the Fleming records and those of the other mediums involved go, it would be naïve skepticism to blindly deny the many incidences of "extraordinary synchronous phenomena" (Hamilton, 2017).

Another of the aristocratic women who formed the core mediums involved in the cross-correspondences was Winifred Coombe-Tennant (then writing under the pseudonym Mrs Willet). She also described her state when automatic writing in terms that are congruent with the above conceptualization. She experienced this state as a division in the conscious self: One part of herself became the communicators but at the same time some form of unity was maintained in the form of a double state of consciousness. Some of this is evident in her words:

Don't you ever walk out of yourself? Aren't you tired of always being yourself? It's so heavenly to be out of myself-when I am everything, and everything else is me .... so strange to be someone else, to feel somebody's heart beating inside you, someone else's mind inside your mind, And there isn't any time or place and either you're loosed or they have entered, and you all of you sudden know everything there ever was. (Quoted in Tyrell 1960, p. 160)

The eminent philosopher C. D. Broad writes in his assessment of the Fleming mediumship that even with a skeptical assessment: "We shall then have to postulate in some stratum of Mrs Willet's mind rather remarkable powers of acquiring information from unread books or the minds of living persons or both" (Broad, 1962, p. 313).

The third major medium, Leonora Piper, involved in the crosscorrespondences showed in contrast a "trance state." This state seemed to be more than role-play, as some modern theories of hypnotic trance attempt to explain this, since she did not respond to various unexpected painful stimuli. She showed signs of a sleep-onset or hypnoidal state merging into what was described as a genuine "possession state" (Sidgwick, 1915), which will be discussed later.

\section{Apparitional and Poltergeist Phenomena}

Apparitional cases are of several different types. The most commonly reported are crisis apparitions occurring synchronously with death or some major emotional event. Given the evidence that 
some of these are non-chance events and not explicable by simple expectancy (Haraldsson, 2012), they can be understood as having a psi component in the sense of expressing the connectedness between the consciousnesses of individuals who have an emotional bond. There is nothing new here and neither is there anything new in the long list of normal explanations for many of these experiences, such as sleep deprivation, stress, fantasy proneness, hypersensitivity, the effect of prior beliefs, and expectancies. Often "normal cases" are used as straw men to dismiss the whole area and to avoid the challenging cases.

One of the classical challenging cases is the Rosenheim case where phones repeatedly dialed by themselves, lamps swung by themselves, electric bulbs burst, pictures and calendars fell off walls, and large objects moved of their own accord (Resch, 1968; Bender, 1971). Normal explanations were excluded as far as humanly possible through the full resources of German meticulousness. These resources included those of the Criminal Investigation Department of the Police, the Municipal Fire Service, the Building Works, and the Electricity Department-as well as physicists from the Max Planck Institute in Munich. There were at least forty persons who were first-hand witnesses to the events. $R$ \& A can perhaps be consoled by how, despite all these efforts, nothing was learned about the causal aspects (other than that a young woman was the focus person).

One of the early documented historical accounts and which despite its age is more revealing concerns the Rerrick case in Scotland from 1695. The case was a mixture of a haunting and poltergeist witnessed by fourteen individuals; five of whom were clergymen (Gauld \& Cornell, 1979). Several witnesses saw apparitions and phantasms along with typical poltergeist phenomena such as stone throwing, raps, fire setting, the inexplicable movement of furniture, and the disturbance of animals. Because poltergeist phenomena are framed in terms of the culture and context of the time, the Rerrick case was readily attributed to demonic crafts. What is revealing in all the well-attested cases is that the phenomena often involved the hurling of heavy objects through the air and the setting of fires, many of which were potentially lethal to those in the vicinity. This does not just apply to the Rerrick case but seems to be a recurrent objective feature of virtually all cases. The skeptic, who obstinately attributes poltergeist phenomena to fraud, would then be 
forced to concede that the perpetrators have a seemingly extraordinary ability, bordering on the magical, to consistently just miss hurting or killing the persons involved. Research psychologist Alan Gauld writes: "These intelligences, which so clearly reflect prevailing folklore and religious beliefs, must simply be aspects of living people ... acting in ways we do not understand" (Gauld, in Gauld \& Cornell, 1979, p. 171).

A recent case which may be further revealing as to the nature of the phenomena, is the South Shields poltergeist (Hallowell \& Ritson, 2009) reviewed by Alan Murdie (2010). The phenomena here included not only classical poltergeists but also more conventional expressions in the form of toys coming to life and threatening text messages being left on mobile phones. The poltergeist was similar to the one in the Rerrick case, responsive and interactive, showing it was not just a set of anomalous phenomena but with its own developed identity. Ironically, despite the modernity, there occurred later a dramatic throwback to medieval times when the amateur investigators became completely convinced that demons, in particular those of jinns, were responsible. Clearly, the case shows how cultural coloring and biased interpretation of the basic phenomena take place. Even cultural repainting can occur. Demon from Greek "daimon" meant originally a godlike being, a lower god, a being between gods and humans, who can be good or bad, and also a spirit of a deceased person (Puhle, 2019).

This sinister aspect of this case shows a resemblance to another contemporary case (Fisher, 1990). Fisher obtained through group séances with a medium the exact details of the former lives the medium's communicators were claiming to have had. Extensive investigation in England and Greece confirmed many of these improbable and virtually inaccessible details. In one such case, that of "Ernest," obscure and personal information concerning his bomber squadron was given and confirmed by still-living members of the squadron, and yet Ernest himself almost certainly never existed. This led Fisher to be fully convinced that demonic spirits which we called "Hungry Ghosts" were deceiving him.

The case known in the psychical research literature as "Conjuring up Philip" is one of the few innovative efforts, which may well give further insight into the sought-after "causal relationships." The case concerned a series of séances in Toronto under the direction of the 
former Cambridge University geneticist George Owen, and his wife Iris Owen (Owen \& Sparrow, 1976). The Owens had the idea to invent a ghost story for the members of a séance group to use in the manner of a psychodrama. The fictional biography was dramatic enough to immerse the group in play-like behavior, and after about a year raps occurred that responded to questions. The pattern of responses seemed to indicate the presence of a communicator who had developed its own will. Although the Owen group was able to record audio and video, their attempts at table levitation and producing raps, the paranormal sources for these, were not fully convincing, at least not to the current writer. However, what supports this approach, as potentially illustrating a principle, are the independent and yet similar findings reported by several others (Batcheldor, 1984; Björkhem, 1994; Pilkington, 2006; Türck, 1945). In particular, the Türck case presents a more (although not entirely) convincing form of photographic documentation. The unifying psi-conducive principle they illustrate is to create a group consensus reality in which the normal constraints over what reality is and what is possible are temporarily suspended (Parker, 2010b).

\section{Creativity}

There is considerable evidence to link creativity and altered states, especially dream states (Barrett, 1993; Zink \& Pietrowsky, 2013). There may well be important links between dissociated states and creativity. Recently, John Foxwell and co-workers (2020) at the University of Durham interviewed writers attending a book festival concerning the autonomy of their characters. Close to two-thirds had heard their characters as voices and one-third experienced the characters as separate from their own thoughts and inner voices. More than half of the respondents had experienced some form of visual presence, and ${ }_{61} \%$ thought their characters had their own agency, often determining how the plot would work out. In this investigation, no questions were apparently included about ESP or transpersonal experiences.

The psychical researcher Hereward Carrington (1920/1996) appeared convinced that such characters as thought-forms could gain an autonomy, and he illustrated this claim with a case of a medium apparently responding to a fictional person that a writer was engaged 
in producing. There is some experimental and anecdotal evidence from various studies that writers and artists are more prone to psychic experiences (e.g., Dalton, 1997; Dinnage, 2008; Holt, 2007; Prince, 1963).

Adam Crabtree, who has worked extensively with psychodrama, presented case histories suggesting:

There seems to be within people a natural ability to take on a personality other than their own and to act from within that assumed personality. They appear actually to become that personality and to some extent leave their own personality behind. (Crabtree, 1985, p. 337)

Crabtree goes on to suppose in a similar manner to what is being proposed here that "one's many selves may retain a certain type of consciousness of their own when not operative in the world" (Crabtree, 1985 , p. 348).

What is also highly relevant here concerns the little-known work of the Finnish psychiatrist Remi Kampman (1976), who discovered, contrary to his own expectations, that school pupils capable of producing secondary personalities during hypnosis were actually clinically healthier and more adaptive than those without secondary personalities.

\section{EXPERIMENTAL PARAPSYCHOLOGY}

The skeptic might now ask, supposing forms of dissociation are a unifying concept in spontaneous phenomena, then how does this relate to ESP performance in the laboratory?

There is considerable evidence that the most successful and efficient experimental designs are those using the so-called freeresponse methods: Among these, the ones using altered states, especially the ganzfeld, have the highest effect size (Storm et al., 2010). Yet, even if we go back to the card-guessing paradigm of J. B. Rhine, his star subjects were described as having been in a "state of 'detachment', 'abstraction', 'relaxation', and the like." Rhine's account described in detail the trance-like appearance of his star scorers during testing (Rhine, 1934/1964, p. 131). Remarkably, what is left un-researched are the strategies used by the sender in ESP experiments. A common, perhaps the most usual, instruction is "to feel in some way at one with 
the receiver," which would be in keeping with what is being proposed here. What does not apparently fit with the notion that ESP is a form of connectedness, are the occasions in which it does seem to be a perceptual ability. Contrary to the portrayal by R \& A of there being only marginal effects, there are occasions of perfect or near-perfect performances with Zener cards and the ganzfeld procedure. Perhaps these spectacular successes are somehow due to the involvement of the experimenter maximizing performance. What I agree with $R$ \& $A$ about is that such maximum effects are the best opportunities for revealing errors or causal relationships.

The wish to keep science squeaky clean from experimenter effects may be a forlorn one both in psychology and parapsychology. Even the experimenter's own psychic experiences may well be "a well-kept secret" that determines the track record of success in psi research and, dare I say this, in other areas (Parker \& Millar, 2014).

One the few important findings from the laboratory concerns the misnomer "psi-missing," which rather than missing is the ability to significantly avoid choosing the target. Although often dismissed by skeptics as an ad hoc finding, there seems to be sufficient consistency and conceptual meaning to regard it as a discovery and one having a reality-import. In other words, it would be the laboratory equivalent of the jinxed ability to consistently make the wrong choice in life situations. Rather than a jinx or even a trickster archetype (Hansen, 2001), it would be argued here that it is sufficient to conclude that this is an expression of the self-defeating needs or self-doubt of the person running amok.

Finally, it may be asked how psychokinesis would fit into this conception? For this it would be necessary to give a central role to willpower, which regrettably is largely today forgotten in psychology. The "Will" was a chapter in William James' classic book Principles of Psychology and formed the cognitive psychology of his successor William McDougal, but the monopoly of contemporary cognitive psychology has led to a will-less motivational psychology. For a while it seemed that Libet's experiments in the 1980s, showing that a preparatory or readiness potential preceded decision-making in the brain and suggesting all behavior was pre-determined by neurology, would ban even motivation as an illusory concept. As is often the case for extreme claims, later findings have questioned this radical conclusion so that 
a belief in free will is still at least defendable (Fifel, 2018). "The Will" is also a concept central to Schopenhauer's philosophy which is being revitalized by the philosopher-physicist Bernard Kastrup (2020).

\section{THE FAILURE OF CONVENTIONAL THEORIES OF ALTERED STATES}

The question is how far can the currently accepted theories be stretched to explain rogue phenomena: Does dissociation itself suffice as an explanation? Dissociation was until recently a diagnostic concept applied mainly within the clinical area for diagnosing multiple personality and for explaining hypnotic phenomena (Kihlstrom, 2013). The re-defining of multiple personality as "dissociated identity disorder," has allowed the concept to find a broader application so that now several scales exist for assessing dissociation as a dimension among the normal population (see Parker, 2015a; 2015b for reviews). For instance, such common occurrences as multi-tasking or engaging in conversation with oneself can be seen as a normal form of dissociative behavior. The theory is that information processing can occur outside the awareness of our central executive self-and can have its own memory registration.

An alternative to the concept of dissociation is "social-cognitive theory," which conceives that behavior, which is inconsistent with the person's self-concept or role, is given socially prescribed explanations. A current illustration of this is found in the way that members of the White House have become masters at explaining away presidential indiscretions in more socially approved terms, for example as "lockerroom banter" rather than un-presidential behavior.

The theory gained much credibility due to an experiment in roleplaying carried out by Nicholas Spanos and his co-workers. Students took on the role of having committed murder and were asked to get in touch with "a hidden part of themselves." This worked to the extent that most of them enacted this role and showed signs of what would be clinically regarded as multiple personality. Spanos et al. $(1985,1994)$ has applied this model to some of the above phenomena ranging from demonic possession and spirit possession, to past life regressions. Spanos does not, however, even attempt to deal with any of the above- 
challenging cases or indeed any challenging cases. In circumventing the concept of "dissociation," Spanos also avoids any discussion of the nature of consciousness that lies behind the various forms of role-playing and delegation, especially ones when the roles become incongruent.

This widespread avoidance of issues has meant that contemporary clinical psychology and psychiatry are ill-equipped to deal with some of the rare but traumatic effects of ego loss that can occur through psychedelic and shamanic experiences or indeed spontaneously. Recently Rachek Aviv (2018) documented the serial disappearances of Hannah Upp, who suffered from dissociated fugue. Hannah's consciousness lost all its sense of identity, which seemed to occur on entering what she described as a mystical state. Periodically, she would then go missing, which finally occurred without her being found. Conventional treatment had apparently nothing to offer her or similar such people.

\section{ROGUE FINDINGS IN NEUROSCIENCE}

This failure to deal with rogue phenomena occurs in other areas of neuroscience. There is the current case of Noah, who, as documented by his neurosurgeon Claire Nicholson, was born with hydrocephalus, leaving a mere $2 \%$ of brain volume. Yet by the age of 4 , this had grown to $80 \%$ with little or no sign of intellectual impairment. Much of this success was attributed to high parental involvement, belief, and care (Noah, 2017).

Neurologist and vocal skeptic Steven Novella (2016) argued concerning a similar case (Feuillet et al., 2007), that when the basic structures are in place they can later become functional when the hydrocephalus is treated and the pressure relieved. This ignores how such pressure in radically squashing the fetal brain would not only compress but grossly deform the brain's delicate structures. To believe otherwise would be the Donald Duck character version of the brain in which Donald is flattened by a streamroller but emerges unscathed. There are further dramatic case accounts that current reductionist neuroscience has been found to be totally inadequate in dealing with (Bolte Taylor, 2008; E. F. Kelly, 2018; Pistorius, 2015). There is also a 
review of the discrepancies between cerebral structures and cognitive functioning published by Nahm, Rousseau, and Greyson (2017).

Finally, it should be added that the evidence indicates consciousness has a primary influence, does not just consist of clinical anecdotes but is prominent in two other as yet un-integrated areas of neuroscience, namely hypnosis and psycho-immunology. Experimental hypnosis has existed for as long as experimental parapsychology, and although there is still a lack of consensus about what hypnosis actually is, no authority would seriously question the dramatic effects of hypnosis as a form of psychological intervention that can alter perception (for example creating hallucinations) and the autonomic nervous system (for example heart rate). Likewise, the effect of expectancy and belief on the strength of the immune system has been well-established for many years (Cloninger, 2004; Ornstein \& Swencionis, 1990).

\section{THE IMPLICATIONS FOR A THEORY OF CONSCIOUSNESS}

It would seem clear from the above review that the current science of cognitive psychology has survived through default, in the absence of a better theory and through the choice to ignore or at best downgrade a multitude of phenomena as anomalies. The range and content of these experiences all are pointers to a radically more dynamic view of consciousness than that conceived by the contemporary theories of cognition. Our normal state of consciousness is focused on a temporary and transient social self while other states have potential access to transpersonally connected consciousness. This is not a new supposition but has featured in the works of several contemporary writers (Braude, 1995; Carter, 2012; Crabtree, 1985; Kelly et al., 2015; Rowan, 1990; Tart, 1986).

What is new in the above presentation is not the role of consciousness itself, but of co-consciousness. Co-consciousness can be defined as the simultaneous operation of two independent systems of mental activity. Stephen Braude (1995) has made an extensive discussion of the term with respect to multiple personality and fugue states. The problem, highlighted in Braude's discussion, is that the terms dissociation and co-consciousness are not mutually exclusive and are not absolute, watertight categories. There are varieties of 
dissociation where the barrier between the "alters" differs, with some "alters" being aware of each other and sharing memories, while for others this is not so. A degree of co-consciousness without much of a memory barrier can be said to occur in multi-tasking, but this contrasts with the more extreme form, which occurs in the many cases of mediumship and automatic writing, some of which were mentioned earlier. In the case of thought-forms, it goes one stage further and it would seem that consciousness has been fully transformed into two or more autonomous self-aware entities.

However, thought-forms are insufficient to explain all the vagaries of the phenomena. For this we need to refer to the major contribution of Stanley Krippner who it can be said has more than anyone else produced order among R \& A's "farrago" phenomena. What we experience in an altered state is according to Krippner decided by the different combinations of dissociation and flow with respect to the degree of control exerted over the altered state. Some of the states, such as those occurring in mediums, are instances of controlled dissociation in that they occur largely to order, whereas in multiple personality and possession the states are uncontrolled. Dissociation and flow are seen by Krippner to be opposing poles of the same dimension. If flow is controlled by rituals such as those in shamanism or in the prescribed use of Ayahuasca, then experience can be an ineffable or a mystical one-an encounter with what Krippner terms the "all-self." How these experiences are received depends of course on the prevailing zeitgeist, so that possession states in the context of paganism was seen by the Catholic Church from the fourteenth century onward as works of the devil (Krippner, 2000; Krippner \& Powers, 1997).

Some fundamental changes will eventually be needed in psychology in order to accommodate these findings, and there are signs of this happening. An example is found in the work of the former CERN-physicist and now also philosopher Bernardo Kastrup, who is responsible for some of the blogs and exchanges in Scientific American. There, in collaboration with the modern interpreters of William James and Frederic Myers, Kastrup argues in strong philosophical terms for the function of consciousness in actively creating consensus reality (Kastrup, Crabtree, \& Kelly, 2018). They write: "the one universal consciousness could, as a result, give rise to many alters with private 
inner lives like yours and ours. As such, we may all be alters-dissociated personalities_of universal consciousness." Years earlier in writing about channeling, John Klimo (1998) arrived at the identical conclusion:

We are all sub- or alter-personalities within one Universal Mind .... The sub-personalities within an individual MPD/DID (or co-conscious) subject usually believe that they possess an identity separate from their host or parent mind. Similarly, ... we sub-personalities of the Universal Mind maintain our dissociated states, relatively unconscious of our deeper identity. (Klimo, 1998, p. 357)

\section{CONCLUSION}

Even if consciousness is conceived of as primary in nature, which is the basic assumption in this theoretical reasoning, it seems to have from birth a biologically built-in organizing function (Stern, 1985; Trevarthen, 2011). It is this organizing function that creates a consensus reality. As such, the normal states of consciousness impose restrictions, the socalled basic limiting principles concerning the temporal and spatial relationships that $\mathrm{R} \& \mathrm{~A}$ defer to. Yet these should not be regarded as holy principles since even these show some cultural influence. The Amondawa are an Amazonian tribe first discovered in 1986 who do not have a concept of time that can be "measured, counted, or talked about in the abstract." Individuals do not age but take on new names, which reflect their life stage and position (University of Portmouth, 2011). It could well be that our own concepts make it difficult to capture psi in a test tube since its presence would invalidate many of these principles organizing consensus reality. It is these limitations that sabotage replication efforts in experimental parapsychology, according to Walter von Lucadou (2017). Von Lucadou's way of luring in these limiting principles is to use correlation matrixes composed of alternative ways that psi can morph. The matrix, by allowing alternative outcomes and by applying statistical corrections for multiple analyses, may become a novel way of resolving the replication issue (Walach et al., 2020). Should one be willing to go further (which I doubt many skeptics or physicists would) and regard these effects as nonlocal correlations in quantum theory (Walach et al., 2014), then these effects can be seen as facilitated by dissociated states. 
Leaving aside the controversy surrounding basic limiting principles and now focusing on the phenomena, the evidence from the above interaction between dissociation and co-consciousness then is that many of the entities (irrespective of whether they are occurring in lucid dreams, psychedelic states, NDEs, or mediumistic states) are from their perspective making entirely authentic claims as to their identity. The entity version of Myers communicating through the medium Trix Fleming really does believe itself to be Myers and may have acquired access to some memories of being so. There is a certain irony in that some of the ideas expressed here about the consciousness of split-off entities were already beginning to be explored by Myers when he was in the living form (Myers, 1903/1975, pp. 36-39). Unfortunately, Myer's ideas never caught on, and psychology continued to operate on a theory of science from Isaac Newton and a theory of neuroscience that has changed little since Johannes Müller in the early nineteenth century.

Some signs of a remedy to this situation are found in the current work of cosmologist Bernard Carr (2015) who has developed a dimensional theory of physics that includes human consciousness, where time perception and mystical and ESP experiences are interrelated. However, it seems obvious that for a theory to give us deeper understanding, a more detailed and integrated explanation linked to mainstream psychological science as well as modern physics needs to be forthcoming.

There are, however, problems with the arguments being put forth above. In addition to the unfinished theoretical aspect, much of the case material is unashamedly anecdotal. There is for instance the case cited by Harvard psychologist George Estabrooks, who was an important contributor to what have become our modern ideas about hypnosis (Estabrooks, 1962, 1971). Estrabrooks claimed to have created the hypnotic equivalent of "the Tulpa." By means of auto-suggestion, he was able to conjure up an imaginary polar bear which apparently developed its own willpower and ran amok chasing nurses and became hard to get rid of (Estabrooks, 1957, pp. 93-94). Like the Conjuring Up Philip case, this one, too, lacks serious and extensive followups.

Skeptics seizing on this will of course ignore the insurmountable logistic problems in allocating the virtually nonexistent resources for studying taboo topics. The irony is that we simply do not know if the 
problems of parapsychology highlighted by skeptics are as they claim, insurmountable, or actually a consequence of skeptics' success in effecting a prohibition of research on the topic.

While it is almost a cosmic joke that both the Philip case and the earlier-mentioned Hungry Ghost case actually occurred in R \& A's backyard, it is nevertheless easy to understand the reluctance of skeptics to indulge in scrutinizing activities, which on the surface seem to be frivolous. The paradox is that we may need to accept, if only on heuristic grounds, that the suspension of the rational waking state seems to be a prerequisite for the phenomena to occur. This does not at all have to mean the abandonment of customary controls exerted by the lead experimenter. It is here that genuine skeptics (adhering to the Greek meaning of skeptikoi) have a vital role to play in promoting critical standards in parapsychology research in order to discover a way forward.

So, is there a way forward? Here recognition is also needed for the real experts on the psychology of deception: the professional illusionists. Contrary to what many skeptics might expect, there are surveys and reports indicating that the large majority of magicians both as a group (Truzzi, 1997) and as practitioners (Hansen, 1990) strongly endorse the existence of genuine paranormal phenomena. Since the surveys were not done for public consumption, mere promotion of the mystique in magic would not seem to explain this. The explanation may well be that the performances succeed in creating a magical atmosphere akin to the Philip case in which belief and reality are temporarily suspended and critical ability is so overwhelmed that the psi-conducive atmosphere that follows sometimes allows "real magic" to happen. This would offer an ideal situation for skeptics to cooperate with magicians who have this conviction and these skills.

The ramifications suggested above would not be complete without giving credit also to Shakespeare whose works are replete with this way of thinking (see Puhle \& Parker-Reed, 2017). This is probably best illustrated in the play Julius Caesar when Brutus in a half-waken state before his final battle sees an apparition and demands "what thou art?" The answer, "Thy evil spirit, Brutus" implies this is not in the normal sense an external demon, but is a dramatic representation in the form of dissociation. While Brutus's own consciousness produced 
the apparition, what follows is that the apparition's consciousness gains an independence from Brutus's mind and has its own will, disturbs the sleep of others, and departs against Brutus' wish, leaving him only with a prophesy of being reunited at death on the battlefield. It is perhaps more hope than prophesy that the thoughts about the issues expressed here will help skeptics and psi-researchers find a productive basis for collaboration in the future.

\section{ACKNOWLEDGMENTS}

The author wishes to thank Trevor Hamilton, Annekatrin Puhle, Nils Wiklund, Nemo Mörck, and Bjorn Sjöden for their helpful comments, and the Electors of the Perrott-Warrick Fund for their support.

\section{REFERENCES}

Aviv, R. (2018, April 2). The edge of eternity/How a young woman lost her identity. New Yorker. https://www.newyorker.com/magazine/2018/04/02

Barrett, D. L. (1993). The "Committee of Sleep": A study of dream incubation for problem solving. Dreaming, 3(2), 115-122.

Barrett, D. L. (1994a). Dreaming as a normal model for multiple personality disorder. Chapter 6 in S. J. Lynn \& J. W. Rhue (Eds.), Dissociation: Clinical and theoretical and research perspectives (pp. 123-135). Guilford.

Barrett, D. L. (1994b). Dreams in dissociative disorders. Dreaming, 4(3), 165-175.

Barrett, D. L. (1995). The dream character as a prototype for the multiple personality alter. Dissociation, 8(3), 61-68.

Barrett, D. L. (1996). Dreams in multiple personality disorder. In D. L. Barrett (Ed.), Trauma and dreams (pp. 74-93). Harvard University Press.

Batcheldor, K. J. (1984). Contributions to the theory of PK induction from sittergroup work. Journal of the American Society for Psychical Research, 78, 105122.

Bender, H. (1971). Modern poltergeist research: A plea for an unprejudiced approach. In J. Beloff (Ed.), New directions in parapsychological research (pp. 122-143). Paul Elek.

Björkhem, O. (1994, August 7-10). A theory of the event horizon. Proceedings of Presented Papers, Parapsychological Association. 37 $7^{\text {th }}$ Annual Convention, Amsterdam, Netherlands, pp. 57-64.

Bolte Taylor, J. (2008). My stroke of insight: A brain scientist's personal journey. Penguin Random House.

Braude, S. E. (1995). First person plural: Multiple personality and the philosophy of mind. Rowman \& Littlefield. 
Braude, S. E. (2003). Immortal remains: The evidence for life after death. Rowman \& Littlefield.

Broad, C. D. (1962). Lectures on psychical research. Routledge \& Kegan Paul.

Brown, D. J. (2016). Dreaming wide awake: Lucid dreaming, shamanic healing, and psychedelics. Park Street Press.

Carpenter, J. C. (1991). Prediction of forced-choice ESP performance: Part III. Three attempts to retrieve coded information using mood reports and a repeated guessing technique. Journal of Parapsychology, 55, 227-280.

Carpenter, J. C. (2012). First sight: ESP and parapsychology in everyday life. Rowman \& Littlefield.

Carr, B. (2015). Hyperspatial modes of matter and mind. In E. F. Kelly, A. Crabtree, \& P. Marshall, (Eds.), Beyond physicalism: Toward reconciliation of science and spirituality (Chapter 7, pp. 227-274). Rowman \& Littlefield.

Carr, B. (2019). Blind watchers of psi: A rebuttal of Reber and Alcock. Journal of Scientific Exploration, 33(4), 643-66o.

Carrington, H. (1920/1996). Your psychic powers and how to develop them. Newcastle Publishing. (Original work published 1920)

Carter, C. (2012). Science and the after-life experience: Evidence for the immortality of consciousness. Inner Traditions.

Cloninger, C. R. (2004). Feeling good: The science of well-being. Oxford University Press.

Cott, C., \& Rock, A. (2008). Phenomenology of N,N-dimethyltryptamine use: A thematic analysis. Journal of Scientific Exploration, 22(3), 359-370.

Crabtree, A. (1985). Multiple man: Explorations in possession and multiple personality. Collins.

Dalton, K. (1997, August 7-10). Exploring the links: Creativity and psi in the Ganzfeld. Proceedings of Presented Papers, Parapsychological Association, 4oth Annual Convention, Brighton, England, pp. 119-134.

Diliberto, G. (2010, September). Patience Worth: Author from the great beyond. Smithsonian Magazine.

Dinnage, R. (2008, October). Psi in the life of writers. Paranormal Review, 3-7.

Dossey, L. (2012). Savants: What they can teach us. EXPLORE, 8(4), 213-217.

Estabrooks, G. H. (1957). Hypnotism (rev. ed.). Dutton \& Co.

Estabrooks, G. H. (1962). Hypnosis: Current problems. Harper \& Row.

Estabrooks, G. H. (1971, April). Hypnosis comes of age. Science Digest, 44-50.

Fenwick, P., Lovelace, H., \& Brayne, S. (2010). Comfort for the dying: Five-year retrospective and one-year prospective studies of end-of-life experiences. Archives of Gerontology and Geriatrics, 51(2), 173-179.

Feuillet, L., Dufour, H., \& Pelletier, J. (2007). Brain of a white-collar worker. Lancet, 370, 262. https://doi.org/10.1016/So140-6736(07)61127-1

Fifel, K. (2018). Readiness potential and neuronal determinism: New insights on Libet Experiment. Journal of Neuroscience, 38(4), 784-786.

https://www.jneurosci.org/content/38/4/784 
Fisher, J. (1990). Hungry ghosts: An investigation into channelling and the spirit world. Harper Collins.

Foxwell, J., Alderson-Day, B., Fernyhough, C., \& Woods, A. (2020). 'I've learned I need to treat my characters like people': Varieties of agency and interaction in writers' experiences of their characters' voices. Consciousness and Cognition, 79, 102901. https://www.sciencedirect.com/science/article/pii/ S1053810019304155

Gauld, A., \& Cornell, A. D. (1979). Poltergeists. Routledge \& Kegan Paul.

Hallowell, M., \& Ritson, D. W. (2009). The South Shields poltergeist: One family's fight against an invisible intruder. The History Press.

Hamilton, T. (2017). Arthur Balfour's ghosts: An Edwardian elite and the riddle of the cross-correspondence automatic writings. Imprint.

Hansen, G. (1990, August). Magicians who endorsed psychic phenomena. The Linking Ring, 70(80), pp. 52-54.

Hansen, G. P. (2001). The trickster and the paranormal. Xlibris.

Haraldsson, E. (2012). The departed among the living: An investigative study of afterlife encounters. White Crow.

Holden, J. M., Greyson, B., \& James, D. (2009). The handbook of near-death experiences: Thirty years of investigation. Praeger, ABC CLIO.

Holt, N. J. (2007, August 2-5). Are artistic populations "psi conducive"? Testing the relationship between creativity and psi with an experience-sampling protocol. Proceedings of Presented Papers, 50th Convention of the Parapsychological Association, Halifax, NS, Canada, pp. 31-47.

Honorton, C. (1977). Psi and internal attention states. In B. Wolman (Ed.)., Handbook of parapsychology. Van Nostrand Reinhold.

Honorton, C., Berger, R. E., Varvoglis, M. P., Quant, M., Derr, P., Schechter, E. I., \& Ferrari, D. C. (1990). Psi communication in the Ganzfeld: Experiments with an automated testing system and a comparison with a meta-analysis of earlier studies. Journal of Parapsychology, 54(2), 99-139.

Johnson, A. (1908-1909). On the automatic writing of Mrs Holland. Proceedings of the Society for Psychical Research, 21, 166-391.

Johnson, C. R. (2017). Complete book of lucid dreaming. Llewellyn Publications.

Kampman, R. (1976). Hypnotically induced multiple personality: An experimental study. International Journal of Clinical and Experimental Hypnosis, 24(3-4), 215-227.

Kastrup, B. (2019). Reasonable inferences from quantum mechanics: A response to "Quantum misuse in psychic literature." Journal of Near-Death Studies, 37(3), 185-200.

Kastrup, B. (2020). Decoding Schopenhauer's metaphysics: The key to understanding how it solves the hard problem of consciousness and the paradoxes of quantum mechanics. Iff Books.

Kastrup, B., Crabtree, A., \& Kelly, E. F. (2018, June 18). Could multiple personality disorder explain life, the universe, and everything? Scientific American. 
https://blogs.scientificamerican.com/observations/could-multiplepersonality-disorder-explain-life-the-universe-and-everything/

Kelly, E. F. (2018). Review of Into the grey zone: A neuroscientist explores the border between life and death by Adrian Owen. Journal of Scientific Exploration, 32(2), 433-445.

Kelly, E. F., Kelly, E. W., Crabtree, A., Grosso, M., \& Greyson, B. (2007). Irreducible mind: Toward a psychology for the 21st century. Rowman \& Littlefield.

Kelly, E. F., Crabtree, A., \& Marshall, P. (2015). Beyond physicalism: Toward a reconciliation of science and spirituality. Rowman \& Littlefield.

Kelly, E. W. (2001). Near-death experiences with reports of meeting deceased people. Death Studies, 25(3), 229-249.

Kihlstrom, J. F. (2013). Neuro-hypnotism: Prospects for hypnosis and neuroscience. Cortex, 49(2), 365-374.

Kirsch, I., \& Council, J. R. (1992). Situational and personality correlates of hypnotic responsiveness. In E. Fromm \& M. R. Nash (Eds.), Contemporary hypnosis research (pp. 267-291). Guilford Press.

Klimo, J. (1998). Channeling: Investigations on receiving information from paranormal sources. North Atlantic Books.

Krippner, S. (2000). A cross-cultural model of dissociation and its inclusion of anomalous phenomena. European Journal of Parapsychology, 15, 3-29.

Krippner, S., \& Powers, S. M. (Eds.). (1997). Broken images, broken selves: Dissociative narratives in clinical practice. Brunner/Mazel.

Luke, D. (2012). Psychoactive substances and paranormal phenomena: A comprehensive review. International Journal of Transpersonal Studies, 31(1), 97-156.

Luke, D. (2017). Otherworlds: Psychedelics and exceptional human experience. Muswell Hill Press.

Marwaha, S. B., \& May, E. C. (2015). Fundamentals issues for psi theorists. In E. C. May \& S. B. Marwaha (Eds.), Extrasensory perception: Support, skepticism, and science, Volume II: Theories of psi. Praeger.

Mikles, N. L., \& Laycock, J. P. (2015). Research Note. Tracking the tulpa. Exploring the "Tibetan" origins of a contemporary paranormal idea. Nova Religio: The Journal of Alternative and Emergent Religions, 19(1), 87-97.

Mroczkowski, J. A., \& Malozemoff, A. P. (2019). Quantum misuse in psychic literature. Journal of Near-Death Studies, 37(3), 131-154.

Murdie, A. (2010). Review of The South Shields poltergeist: One family's fight against an invisible intruder by M. J. Hallowell and D. W. Ritson. Journal of the Society for Psychical Research, 73(2), 129-132.

Murphy, G. (1966). Research in creativeness: What can it tell us about extrasensory perception? Journal of the American Society for Psychical Research, 6o, 8-22.

Myers, F. W. H. (1975). Human personality and its survival of bodily death (Vol. 1). Arno. (Original work published in 1903)

Nahm, M. (2011). Reflections on the context of near-death experiences. Journal of Scientific Exploration, 25(3), 453-478. 
Nahm, M., Rousseau, D., \& Greyson, B. (2017). Discrepancy between cerebral structures and cognitive functioning: A review. Journal of Nervous and Mental Disease, 205(12), 967-972.

Noah. (2017). The boy with no brain: Extraordinary people. Channel 4 Production. https://www.youtube.com/watch?v=YeZeXb-MY-s

Novella, S. (2016). Man living with $10 \%$ of his brain? Neurologica blog. https:// theness.com/neurologicablog/index.php/man-living-with-1o-of-his-brain/

Ornstein, R., \& Swencionis, C. (Eds.). (1990). The healing brain: A scientific reader. Guilford Press.

Owen, I., \& Sparrow, M. (1976). Conjuring up Phillip: An adventure in psychokinesis. Harper \& Row.

Parker, A. (1975). Some findings relevant to the change in state hypothesis. In J. D. Morris, W. G. Roll, \& R. L. Morris (Eds.). Research in Parapsychology 1974 (pp. 40-42). Scarecrow Press.

Parker, A. (1994, August 7-10). Eyeballing the ganzfeld. Proceedings of Presented Papers Parapsychological Association, 37th Annual Convention, Amsterdam, Netherlands, p. 314.

Parker, A. (2000). A review of the Ganzfeld work at Gothenburg University. Journal of the Society for Psychical Research, 64, 1-15.

Parker, A. (2010a). Review of The eager dead by Archie Roy. Journal of Scientific Exploration, 24(3), 546-553.

Parker, A. (2010b). The mind-body problem and the issue of psychokinesis. In S.

Krippner and H. L. Friedman (Eds.), Mysterious minds: The neurobiology

of psychics, mediums, and other extraordinary people. Praeger.

Parker, A. (2011). Review of Born on a blue day \& Embracing the wide sky by Daniel Tammet. Journal of Scientific Exploration, 25(4), 841-848.

Parker, A. (2015a). The jungle of hypnotic psi: Part 1 . Research on hypnosis relevant to psi. Journal of Parapsychology, 79(1), 20-36.

Parker, A. (2015b). The jungle of hypnotic psi: Part 2. Research on relationships between psi and hypnosis. Journal of Parapsychology, 79(1), 37-52.

Parker, A. (2017, September 1-3). Current research on lucid dreaming and shared lucidity states. Proceedings of $41^{\text {st }}$ International Conference of the Society for Psychical Research, Horsely Estate, United Kingdom. https://gup.ub.gu.se/ publication $/ 276730$

Parker, A. (2020). Contribution in D. Marks, Psychology and the paranormal: Exploring anomalous experience. Sage.

Parker, A., \& Millar, B. (2014). Revealing psi secrets: Successful experimenters seem to succeed by using their own psi. Journal of Parapsychology, 78(1), 39-55.

Parker, A., \& Puhle, A. (2018). 'Thoughtforms'. Psi Encyclopedia. The Society for Psychical Research. https://psi-encyclopedia.spr.ac.uk/articles/ thoughtforms

Parker, A., Frederiksen, A., \& Johansson, H. (1997). Towards specifying the recipe for success with the Ganzfeld: Replication of the Ganzfeld findings using 
a manual Ganzfeld with subjects reporting paranormal experiences.. European Journal of Parapsychology, 13, 15-27.

Parker, A., Persson, A., \& Haller, A. (2000). Using qualitative Ganzfeld research for theory development: Top-down processes in psi-mediation. Journal of the Society for Psychical Research, 64, 65-81.

Pilkington, R. (2006). The spirit of Dr. Bindelof: The enigma of séance phenomena. Anomalist Books.

Pilotti, J. (2011). Consciousness and physics: Towards a scientific proof that consciousness is in space-time beyond the brain. Journal of Transpersonal Research, 3(2), 123-134.

Pistorius, M. (2015). Ghost boy: The miraculous escape of a misdiagnosed boy trapped inside his own body. Simon \& Schuster.

Prince, W. F. (1964). The case of Patience Worth. University Books. (Original work 1927)

Prince, W. F. (1963). Noted witnesses for psychic occurrences. University Books.

Puhle, A. (2019). Mit Goethe durch die Welt der Geister. Reichl Rörlag, 2, 470. http://www.blumhardt-online.de/reichl_puhle.html

Puhle, A., \& Parker, A. (2017). An exploratory study of lucid dreams concerning deceased persons. Journal of the Society for Psychical Research, 81(3), 145-160.

Puhle, A., \& Parker-Reed, A. (2017). Shakespeare's ghosts live: From Shakespeare's ghosts to modern psychical research. Cambridge Scholars Publishing.

Radin, D. I. (2013). Supernormal: Science, yoga, and the evidence for extraordinary psychic abilities. Deepak Chopra Books.

Radin, D. I. (2018). Real magic: Ancient wisdom, modern science, and a guide to the secret power of the universe. Harmony Books.

Reber, A. S., \& Alcock, J. E. (2020). Searching for the impossible: Parapsychology's elusive quest. American Psychologist, 75(3), 391-399.

Resch, A. (1968). Der Fall Rosenheim (I/II/III). Grenzgebiete der Wissenschaft, 17, 241249, 289-310, 337-346.

Rhine, J. B. (1964). Extrasensory perception. Bruce Humphries. (Original work published 1934)

Rivas, T., Dirven, A., \& Smit, R. H. (2016). The self does not die: Verified paranormal phenomena from near-death experiences. International Association for NearDeath Studies.

Roe, C. A. (2019). The role of theory in psychical research. Paranormal Review, 91, 4-5.

Rowan, J. (1990). Subpersonalities: The people inside us. Routledge.

Schredl, M., \& Erlacher, D. (2004). Lucid dreaming frequency and personality. Personality and Individual Differences, 37(7), 1463-1473.

Sheldrake, R. (2020). Contribution in D. Marks (Ed.), Psychology and the paranormal: Exploring anomalous experience. Sage.

Sidgwick, E. (1915). A contribution to the study of the psychology of Mrs Piper's 
trance phenomena. Proceedings of the Society for Psychical Research, 28, $1-652$.

Spanos, N. P. (1994). Multiple identity enactments and multiple personality disorder: A sociocognitive perspective. Psychological Bulletin, 116(1), 143-165. https://doi.org/10.1037/0033-2909.116.1.143

Spanos, N. P., Weekes, J. R., \& Bertrand, L. D. (1985). Multiple personality: A social psychological perspective. Journal of Abnormal Psychology, 94(3), 362-376.

Stapp, H. P. (2015). A quantum-mechanical theory of the mind/brain connection. In E. F. Kelly, A. Crabtree, \& P. Marshall (Eds.), Beyond physicalism: Toward reconciliation of science and spirituality (Chapter 5, pp. 157-194). Rowman \& Littlefield.

Stern, D. N. (1985). The interpersonal world of the infant: A view from psychoanalysis and development psychology. Basic Books.

Storm, L., Tressoldi, P. E., \& Di Risio, L. (2010). Meta-analysis of free-response studies, 1992-2008: Assessing the noise reduction model in parapsychology. Psychological Bulletin, 136(1), 471-485.

Strassman, R. (2001). DMT: The spirit molecule: A doctor's revolutionary research into the biology of near-death and mystical experiences. Park Street Press.

Stumbrys, T., \& Daniels, M. (2010). An exploratory study of creative problem solving in lucid dreams: Preliminary findings and methodological considerations. International Journal of Dream Research, 3(2), 121-129.

Stumbrys, T., \& Erlacher, D. (2017). Inner ghosts: Encounters with threatening dream characters in lucid dreams. Dreaming, 27(1), 40-48.

Stumbrys, T., Erlacher, D., \& Schmidt, S. C. E. (2011). Lucid dream mathematics: An explorative online study of arithmetic abilities of dream characters. International Journal of Dream Research, 4(1), 35-40.

Tart, C. T. (1986). Waking up: Overcoming the obstacles to human potential. Element Books.

Tholey, P. (1989). Overview of the development of lucid dream research in Germany. http://gestalttheory.net/archive/thol_lucidı.html

Treffert, D. A. (2000). Extraordinary people: Understanding savant syndrome. iUniverse.

Treffert, D. A. (2009). The savant syndrome: An extraordinary condition. A synopsis: Past, present, future. Philosophical Transactions of the Royal Society B, 364(1522), 1351-1357. https://doi.org/10.1098/rstb.2008.0326

Treffert, D. A. (2010). Islands of genius: The bountiful mind of the autistic, acquired, and sudden savant. Jessica Kingsley.

Treffert, D. A. (2017). Accidental genius. https://www.youtube.com/ watch? $\mathrm{v}=$ WxeıPkyjev8

Trevarthen, C. (2011). What is it like to be a person who knows nothing? Defining the active intersubjective mind of a newborn human being. Infant and Child Development, 20, 119-135. 10.1002/icd.689 
Truzzi, M. (1997). Reflections on the sociology and social psychology of conjurors and their relations with psychical research. In S. Krippner (Ed.), Advances in parapsychological research. McFarland.

Türck, S. (1945). Jeg var Dus med Aanderne [I got personal with the spirits]. Hasselbalch.

Tyrrell, G. N. M. (1960). The personality of man: New facts and their significance. Penguin Books.

University of Portsmouth. (2011, May 20). 'Time' not necessarily deeply rooted in our brains. Medical press psychology/psychiatry. https://medicalxpress.com/ news/2011-05-necessarily-deeply-rooted-brains.html

van Lommel (2020). Quantum physics as analogy: A response to "Quantum Misuse in Psychic Literature." Journal of Near-Death Studies, 37(3), 174-184.

von Lucadou, W. (2017). Clinical parapsychology. Proceedings of the Norwegian Society for Psychical Research, 84, 57-63.

Waggoner, R., \& McCready, C. (2015). Lucid dreaming: Plain and simple. Conari Press.

Walach, H., Horan, M., Hinterberger, T., \& von Lucadou, W. (2020). Evidence for anomalistic correlations between human behavior and a random event generator: Result of an independent replication of a micro-PK experiment. Psychology of Consciousness: Theory, Research, and Practice, 7(2), 173-188.

Walach, H., von Ludacou, W., \& Römer, H. (2014). Parapsychological phenomena as examples of generalized non-local correlations-A theoretical framework. Journal of Scientific Exploration, 28(4), 605-631.

Williams, B. J. (2019). Reassessing the "Impossible": A critical commentary on Reber and Alcock's "Why parapsychological claims cannot be true." Journal of Scientific Exploration, 33(4), 599-616.

Zink, N., \& Pietrowsky, R. (2013). Relationship between lucid dreaming, creativity, and dream characteristics. International Journal of Dream Research, 6(2), 98-103. 